\title{
Abundance analysis of red clump stars in the old, inner disc, open cluster NGC 4337: a twin of NGC 752?`
}

\author{
Giovanni Carraro $^{1, \star \star}$, Lorenzo Monaco ${ }^{1}$, and Sandro Villanova ${ }^{2}$
}

\author{
${ }^{1}$ ESO, Alonso de Cordova 3107, 19001 Santiago de Chile, Chile \\ e-mail: [gcarraro,lmonaco]@eso.org \\ 2 Departamento de Astronomía, Universidad de Concepción, Casilla 169, Concepción, Chile \\ e-mail: svillanova@astro-udec.cl
}

Received 20 June 2014 / Accepted 16 July 2014

\begin{abstract}
Context. Open star clusters older than $\sim 1$ Gyr are rare in the inner Galactic disc. Still, they are objects that hold crucial information for probing the chemical evolution of these regions of the Milky Way.

Aims. We aim at increasing the number of old open clusters in the inner disc for which high-resolution metal abundances are available. Here we report on NGC 4337, which was recently discovered to be an old, inner disc open cluster.

Methods. We present the very first high-resolution spectroscopy of seven clump stars that are all cluster members. We performed a detailed abundance analysis for them.

Results. We find that NGC 4337 is marginally more metal-rich than the Sun, with $[\mathrm{Fe} / \mathrm{H}]=+0.12 \pm 0.05$. The abundance ratios of $\alpha$-elements are generally solar. At odds with recent studies on intermediate-age and old open clusters in the Galactic disc, Ba is under-abundant in NGC 4337 compared with the Sun. Our analysis of the iron-peak elements $(\mathrm{Cr}$ and Ni) does not reveal anything anomalous. Based on these results, we estimate the cluster age to be $1.6_{-0.1}^{+0.1} \mathrm{Gyr}$, and derive a reddening $E(B-V)=0.23 \pm 0.05$, and an apparent distance modulus $(m-M)_{V}=12.80 \pm 0.15$. Its distance to the Galactic centre is $7.6 \mathrm{kpc}$. With this distance and metallicity, NGC 4337 fits the metallicity gradient for the inner Galactic disc fairly well.

Conclusions. The age and metallicity we measured make NGC 4337 a twin of the well-known old open cluster NGC 752 . The red clumps of these two clusters bear an amazing resemblance. But the main sequence of NGC 752 is significantly more depleted in stars than that of NGC 4337. This would mean that NGC 752 is in a much more advanced dynamical stage, being on the verge of dissolving into the general Galactic field. Our results make NGC 4337 an extremely interesting object for further studies of stellar evolution in the critical turn-off mass range 1.1-1.4 $M_{\odot}$.
\end{abstract}

Key words. techniques: spectroscopic - Hertzsprung-Russell and C-M diagrams - open clusters and associations: general open clusters and associations: individual: NGC 4337

\section{Introduction}

Recently, Carraro et al. (2014a) have drawn the attention to the open star cluster NGC 4337, located at $l=299^{\circ} .3, b=+4^{\circ} .6$. It was identified as a particularly relevant object, being a rare example of an old ( 1.5-2.0 Gyr) cluster located inside the solar ring at a Galacto-centric distance $R_{\mathrm{GC}} \sim 7.5 \mathrm{kpc}$. As such, NGC 4337 can help us to improve our knowledge of the inner disc radial abundance gradient and its temporal evolution (Magrini et al. 2010, 2014).

This evidence came from the analysis of a deep photometric data-set in the $U, B, V$, and $I$ passbands, and using the classical old open IC 4651 (Anthony-Twarog et al. 2009) ridge-line as comparison. Previous shallow photoelectric optical photometry had missed the cluster, but more recently, it was recovered by Froebrich et al. (2010) in their search for star clusters across the Galactic plane using 2MASS.

Carraro et al. (2014a) assumed that NGC 4337 shares the same metallicity as IC 4651 from simply comparing the

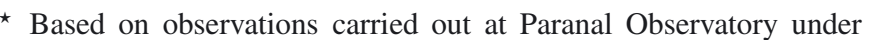
program 292.D-5043.

$\star \star$ On leave from Dipartimento di Fisica e Astronomia, Universitá di Padova, Italy.
}

distribution of stars in the colour-magnitude diagram (CMD). Based on this, preliminary estimates of the reddening $(E(B-$ $V) \sim 0.26)$ and the apparent distance modulus $\left((m-M)_{V} \sim\right.$ 13.00) were derived.

With this preliminary fundamental parameter set, NGC 4337 is shown to be an extremely interesting object, sharing the same age and hence turn-off (TO) mass as IC 4651, NGC 3680, and NGC 752. These text-book clusters have routinely been studied to probe the behaviour of H-burning in the TO mass range 1.1-1.4 $M_{\odot}$, where convective overshooting is expected to take place. Because NGC 4337 is much richer in stars than these clusters, it is an ideal target to compare its CMD with stellar models (Bertelli et al. 1992; Carraro et al. 1993, 1994). The NGC 4337 clump is also particularly rich and well defined, allowing for direct comparisons with stellar models of the He-burning phase in this critical mass range (Girardi et al. 2000).

In this paper we follow the study of Carraro et al. (2014a) up and present for the first time high-resolution spectroscopy obtained with the FLAMES-UVES spectrograph at ESO Paranal. These data are used to measure the abundance of several elements (such as $\alpha$, neutron-capture, and iron peak) for the stars in the red clump of NGC 4337. The knowledge of the metal abundance, in turn, allows us to provide more solid estimates of the 
Table 1. Clump stars observed with UVES.

\begin{tabular}{lccrrrr}
\hline \hline ID & RV & RA(2000.0) & Dec(2000.0) & $V$ & $(B-V)$ & $(V-I)$ \\
\hline & $\mathrm{km} \mathrm{s}^{-1}$ & hh:mm:ss.sss & dd:mm:ss.ss & mag & mag & \\
\hline 90 & $-17.779 \pm 1.046$ & $12: 24: 15.761$ & $-58: 08: 50.17$ & 13.80 & 1.27 & 1.31 \\
91 & $-18.180 \pm 1.190$ & $12: 24: 39.259$ & $-58: 07: 49.69$ & 13.83 & 1.31 & 1.40 \\
99 & $-18.311 \pm 1.026$ & $12: 24: 39.482$ & $-58: 13: 20.24$ & 13.85 & 1.30 & 1.41 \\
100 & $-17.898 \pm 1.227$ & $12: 24: 00.948$ & $-58: 05: 51.22$ & 13.86 & 1.33 & 1.37 \\
102 & $-15.976 \pm 1.102$ & $12: 23: 27.228$ & $-58: 02: 50.24$ & 13.86 & 1.35 & 1.45 \\
115 & $-18.224 \pm 1.102$ & $12: 23: 56.662$ & $-58: 07: 25.21$ & 13.93 & 1.32 & 1.36 \\
128 & $-17.920 \pm 1.127$ & $12: 24: 44.945$ & $-58: 02: 09.96$ & 14.06 & 1.33 & 1.44 \\
\hline
\end{tabular}

Notes. Coordinates for 2000.0 equinox are reported together with photometry from Carraro et al. (2014a) and heliocentric radial velocities.

cluster fundamental parameters (mostly age and distance), and discuss its properties in the framework of the Milky Way structure and chemical evolution.

The paper is organised as follows: in Sect. 2 we present the observational material and describe how radial velocities are derived. Section 3 is devoted to the abundance analysis, while in Sect. 4 we discuss the results of our analysis in detail. Section 5 summarises our findings.

\section{Observations and data reduction}

Observations were taken in service mode on the night of March 30, 2014 using the multi-object fibre-fed FLAMES facility mounted at the ESO-VLT/UT2 telescope at the Paranal Observatory (Chile). Two 2775 s exposures were taken simultaneously with the GIRAFFE medium-resolution spectrograph, and the red arm of the UVES high-resolution spectrograph. GIRAFFE was configured in Medusa mode in the setup HR15N, which covers the wavelength range 6470-6790 $\AA$ at a resolution of $R=17000$. This set-up was chosen because it simultaneously includes the $\mathrm{Li}$ resonance doublet and the $\mathrm{H}_{\alpha}$ line. The analysis of this data-set will be presented in a forthcoming paper.

The UVES spectrograph was, instead, set up around a $5800 \AA$ central wavelength, thus covering the 4760-6840 wavelength range and providing a resolution of $R \simeq 47000$.

Here we discuss the UVES data. The UVES fivers were placed on probable red-clump targets, and are shown on top of the cluster's $V$ vs. $V-I$ CMD in Fig. 1. Table 1 presents the target star IDs, coordinates, and $B, V$, and $I$ photometry from Carraro et al. (2014a). The data were reduced using the ESO CPL based FLAMES-UVES pipeline version 5.3.0 ${ }^{1}$ to extrac the individual fibre spectra.

The spectra were eventually normalised using the standard IRAF task continuum. Radial velocities were computed using the IRAF/fxcor task to cross-correlate the observed spectra with a synthetic spectrum from the library of Coelho et al. (2005) with stellar parameters $T_{\text {eff }}=4750 \mathrm{~K}, \log g=2.0$, solar metallicity, and no $\alpha$-enhancement. The IRAF rvcorrect task was used to calculate the correction from geocentric velocities to heliocentric.

The seven stars observed with UVES have the same heliocentric radial velocity within the uncertainty (see Table 1), supporting the idea that they are all cluster members. Therefore, the mean heliocentric radial velocity of NGC $4337 \mathrm{RV}=-17.75 \pm$ $0.81 \mathrm{~km} \mathrm{~s}^{-1}$.

\footnotetext{
$\overline{1 \text { http://www.eso.org/sci/software/pipelines/ }}$
}

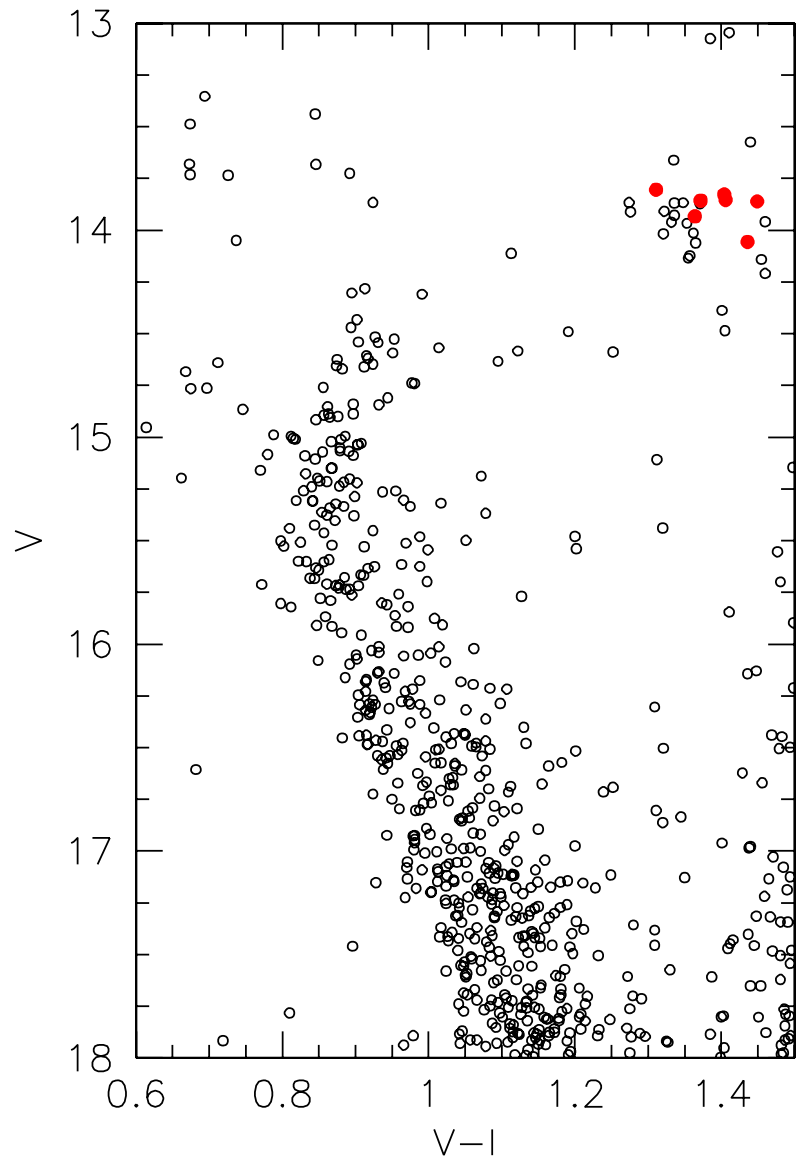

Fig. 1. Colour-magnitude diagram of NGC 4337. Red filled circles identify the seven red clump stars observed with UVES.

\section{Abundance analysis}

The chemical abundances for $\mathrm{Na}, \mathrm{Mg}, \mathrm{Al}, \mathrm{Si}, \mathrm{Ca}, \mathrm{Ti}, \mathrm{Cr}, \mathrm{Fe}$, and $\mathrm{Ni}$ were obtained using the equivalent widths of species transition lines (EW method, as detailed in Marino et al. 2008). For $\mathrm{C}, \mathrm{N}, \mathrm{O}, \mathrm{Y}, \mathrm{Ba}, \mathrm{La}$, and Eu, whose lines are affected by blending, we used the spectrum-synthesis method. For this purpose we calculated five synthetic spectra with different abundances for the elements, and estimated the best-fitting value as the one that minimises the rms scatter. Only lines un-contaminated by telluric lines were used. ATLAS9 (Kurucz 1970) model atmospheres were used for the EW and spectrum-synthesis methods. The initial atmospheric parameters for the model atmosphere were assumed to be those typical for an RGB star of an open cluster, that is $T_{\mathrm{eff}}=4500 \mathrm{~K}, \log g=2.5, v_{\mathrm{t}}=$ $1.20 \mathrm{~km} \mathrm{~s}^{-1}$, and $[\mathrm{Fe} / \mathrm{H}]=0.0$. We then refined them during the 
Table 2. Iron abundance and photospheric parameters for clump stars in NGC 4337.

\begin{tabular}{lrrrr}
\hline \hline ID & $T_{\text {eff }}$ & $\log (g)$ & {$[\mathrm{Fe} / \mathrm{H}]$} & $v_{t}$ \\
\hline \multicolumn{5}{r}{$\mathrm{K}$} \\
\hline 0 & 4790 & 2.65 & +0.10 & 1.19 \\
91 & 4730 & 2.70 & +0.12 & 1.21 \\
99 & 4730 & 2.70 & +0.13 & 1.19 \\
100 & 4760 & 2.60 & +0.09 & 1.27 \\
102 & 4720 & 2.60 & +0.11 & 1.20 \\
115 & 4760 & 2.70 & +0.14 & 1.17 \\
128 & 4720 & 2.55 & +0.13 & 1.13 \\
\hline
\end{tabular}

abundance analysis. As a first step, atmospheric models were calculated using ATLAS9 (Kurucz 1970) with the initial estimates of $T_{\text {eff }}, \log g, v_{\mathrm{t}}$, and $[\mathrm{Fe} / \mathrm{H}]$.

The values of $T_{\text {eff }}, v_{\mathrm{t}}$, and $\log g$ were adjusted and new atmospheric models calculated in an interactive way to remove trends in excitation potential (EP) and equivalent width $v s$. abundance for $T_{\text {eff }}$ and $v_{\mathrm{t}}$, respectively, and to satisfy the ionisation equilibrium for $\log g$. Fe I and Fe II were used for this purpose. The $[\mathrm{Fe} / \mathrm{H}]$ value of the model was changed at each iteration according to the output of the abundance analysis (see Table 2). The local thermodynamic equilibrium (LTE) program MOOG (Sneden 1973) was used for the abundance analysis.

In all the analysis, we closely followed Carraro et al. (2014b). We therefore refer to this study for additional details. We briefly recall that typical internal errors are $\Delta\left(T_{\text {eff }}\right)=50 \mathrm{~K}$, $\Delta \log g=0.2 \mathrm{dex}, \Delta v_{\mathrm{t}}=0.10 \mathrm{~km} \mathrm{~s}^{-1}$, and $\Delta[\mathrm{Fe} / \mathrm{H}]=0.05 \mathrm{dex}$.

The line lists for the chemical analysis were obtained from many sources (Gratton et al. 2003, VALD \& NIST ${ }^{2}$; McWilliam \& Rich 1994; McWilliam 1998, SPECTRUM $^{3}$, and $\mathrm{SCAN}^{4}$ ), and the $\log$ gf were calibrated using the solar-inverse technique and by the spectral synthesis method, as discussed in full detail by Villanova et al. (2009). For this purpose we used the high-resolution, high signal-to-noise ratio NOAO solar spectrum (Kurucz et al. 1984). The solar abundances we obtained with our line list are reported in Table 4, together with those given by Grevesse \& Sauval (1998) for comparison. We emphasise that all the line-lists were calibrated on the Sun, including those used for the spectral synthesis. We provided the line list as a long table in Carraro et al. (2014b). In addition, the $C$ content was obtained from the $\mathrm{C}_{2}$ system at $563.2 \mathrm{~nm}$, and $\mathrm{N}$ from the $\mathrm{CN}$ lines at $634 \mathrm{~nm}$.

Abundances for $\mathrm{C}, \mathrm{N}$, and $\mathrm{O}$ were determined all together in an interactive way to take into account any possible molecular coupling of these three elements. Our targets are objects evolved off the main sequence, so some evolutionary mixing is expected. This can affect the primordial $\mathrm{C}$ and $\mathrm{N}$ abundances separately, but not the total $\mathrm{C}+\mathrm{N}+\mathrm{O}$ content because these elements are transformed one into the other during the $\mathrm{CNO}$ cycle.

\section{Results of abundance analysis}

In this section, we discuss in detail the outcome of the abundance analysis and its impact on the determination of the cluster parameters.

\footnotetext{
2 http://physics.nist.gov/PhysRefData/ASD/

3 http://www. phys.appstate.edu/spectrum/spectrum.html

4 http://www.astro.ku.dk/ uffegj/
}

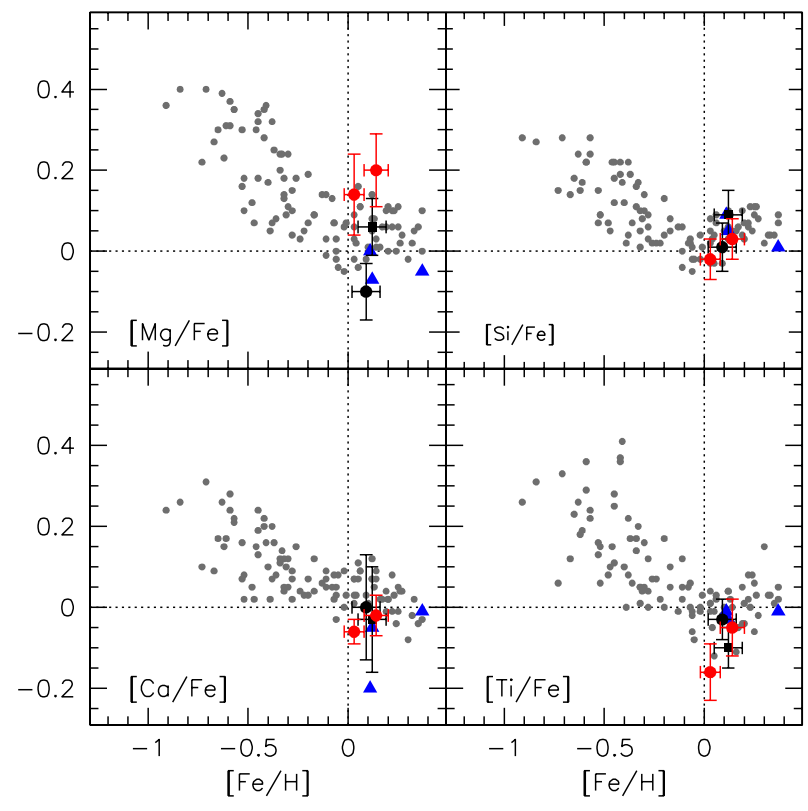

Fig. 2. Comparison between NGC 4337 mean $\alpha$-element ratios (black solid square) including error bars derived from Table 5, with data from old open clusters in the inner disc from Magrini et al. (2010; blue triangles) and inner disc giants from Bensby et al. (2010; grey dots). The black solid circle indicates Trumpler 20 from Carraro et al. (2014b), and the red circles are NGC 4815 and NGC 6705 from Magrini et al. (2014).

\subsection{Metallicity and $\alpha$ elements}

Table 2 lists the metallicity, measured as $[\mathrm{Fe} / \mathrm{H}]$, of the seven clump stars we observed with UVES. The mean value is $[\mathrm{Fe} / \mathrm{H}]=0.12 \pm 0.01$, with very small dispersion (see also Table 4). This is the very first estimate of the metallicity of NGC 4337 , and the value we obtained is well within the expectations for a cluster located inside the solar ring (Magrini et al. 2010, 2014). It is indeed quite similar to Trumpler 20 (Carraro et al. 2014b; Magrini et al. 2014), an open cluster located very close to NGC 4337.

In Fig. 2 we plot the trend of averaged $\alpha$-element abundance of NGC 4337 versus metallicity, an compare them with different objects, The comparison is against $\mathrm{K}$ giants in the inner disc taken from Bensby et al. (2010) study. Besides, we also show a few inner disc open clusters: Trumpler 20 (Carraro et al. 2014b), NGC 4815 and NGC 6705 (Magrini et al. 2014), and NGC 6404, NGC 6503, and NGC 6192 (Magrini et al. 2010). Solar abundances are indicated with dotted lines.

The agreement is in general very good, and all these clusters appear to be members of the same stellar population. The exception is $[\mathrm{Mg} / \mathrm{Fe}]$, which shows a quite significant scatter. This was also reported present in Magrini et al. (2014), because Trumpler 20 shows a $\mathrm{Mg}$ under-abundance with respect to the Sun, similar to the clusters studied in Magrini et al. (2010). NGC 4337, instead, comfortably lies in the general trend defined by $\mathrm{K}$ giants. This is true not only for $\mathrm{Mg}$, but for all the other $\alpha$-elements.

\subsection{Iron peak elements}

We culled from the literature the abundances of thin- and thickdisc stars iron peak element (Ni and Cr, Reddy et al. 2003, 2006) and compare them with our results for NGC 4337 in Fig. 3. We 
Table 3. Individual abundances for NGC 4337 members.

\begin{tabular}{lrrrrrrrrrrrr}
\hline \hline $\mathrm{ID}$ & $\mathrm{C}$ & $\mathrm{N}$ & $\mathrm{O}$ & $\mathrm{CNO}$ & $\mathrm{Na}$ & $\mathrm{Mg}$ & $\mathrm{Al}$ & $\mathrm{Si}$ & $\mathrm{Ca}$ & $\mathrm{Ti}$ & $\alpha$ \\
\hline 90 & -0.14 & 0.26 & -0.03 & -0.02 & 0.33 & 0.10 & 0.12 & 0.13 & -0.02 & -0.12 & 0.00 & \\
91 & -0.16 & 0.33 & -0.01 & -0.00 & 0.32 & 9.99 & 0.15 & 0.13 & -0.05 & -0.13 & -0.02 \\
99 & -0.18 & 0.35 & 0.02 & 0.01 & 0.39 & 0.01 & 0.12 & 0.09 & 0.00 & -0.06 & 0.01 \\
100 & -0.12 & 0.33 & -0.07 & -0.03 & 0.34 & 0.05 & 0.20 & 0.07 & -0.04 & -0.12 & -0.03 \\
102 & -0.14 & 0.32 & -0.05 & -0.02 & 0.36 & 0.10 & 0.18 & 0.06 & -0.05 & -0.07 & -0.02 & \\
115 & -0.15 & 0.27 & -0.06 & -0.04 & 0.33 & 0.05 & 0.19 & 0.07 & -0.02 & -0.06 & 0.00 & \\
128 & -0.19 & 0.29 & -0.12 & -0.08 & 0.34 & 0.04 & 0.17 & 0.07 & $\mathrm{~b} 0.00$ & -0.16 & -0.03 & $\mathrm{Eu}$ \\
\hline \hline $\mathrm{ID}$ & $\mathrm{V}$ & $\mathrm{Cr}$ & $\mathrm{Fe}$ & $\mathrm{Co}$ & $\mathrm{Ni}$ & $\mathrm{Cu}$ & $\mathrm{Zn}$ & $\mathrm{Y}$ & $\mathrm{Zr}$ & $\mathrm{Ba}$ & $\mathrm{Ce}$ & $\mathrm{Eu}$ \\
\hline 90 & 0.58 & -0.02 & 0.10 & 0.24 & 0.09 & 0.12 & 9.99 & -0.26 & 9.99 & -0.06 & -0.25 & 0.08 \\
91 & 0.59 & -0.01 & 0.12 & 0.25 & 0.11 & 0.03 & 9.99 & -0.18 & -0.33 & -0.09 & -0.23 & 0.14 \\
99 & 0.52 & -0.06 & 0.13 & 0.18 & 0.11 & 0.15 & -0.36 & -0.23 & 9.99 & -0.02 & -0.38 & 0.16 \\
100 & 0.60 & 0.02 & 0.09 & 0.28 & 0.10 & 0.09 & -0.38 & -0.23 & -0.30 & -0.16 & 9.99 & 0.09 \\
102 & 9.99 & -0.05 & 0.11 & 0.25 & 0.12 & 0.10 & -0.49 & -0.21 & -0.39 & -0.07 & -0.20 & 0.05 \\
115 & 0.58 & -0.02 & 0.14 & 0.26 & 0.13 & 0.16 & -0.35 & -0.03 & -0.49 & -0.04 & -0.23 & 0.12 \\
128 & 0.48 & -0.04 & 0.13 & 0.29 & 0.14 & 0.10 & 9.99 & -0.33 & 9.99 & -0.15 & -0.25 & -0.01 \\
\hline
\end{tabular}

Notes. In addition to iron, presented as $[\mathrm{Fe} / \mathrm{H}]$, abundances are provided as $[\mathrm{X} / \mathrm{Fe}]$, where $\mathrm{X}$ is the chemical species. With $\alpha$ we indicate the average of the $\alpha$-elements $\mathrm{O}, \mathrm{Mg}, \mathrm{Si}, \mathrm{Ca}$, and Ti abundances.

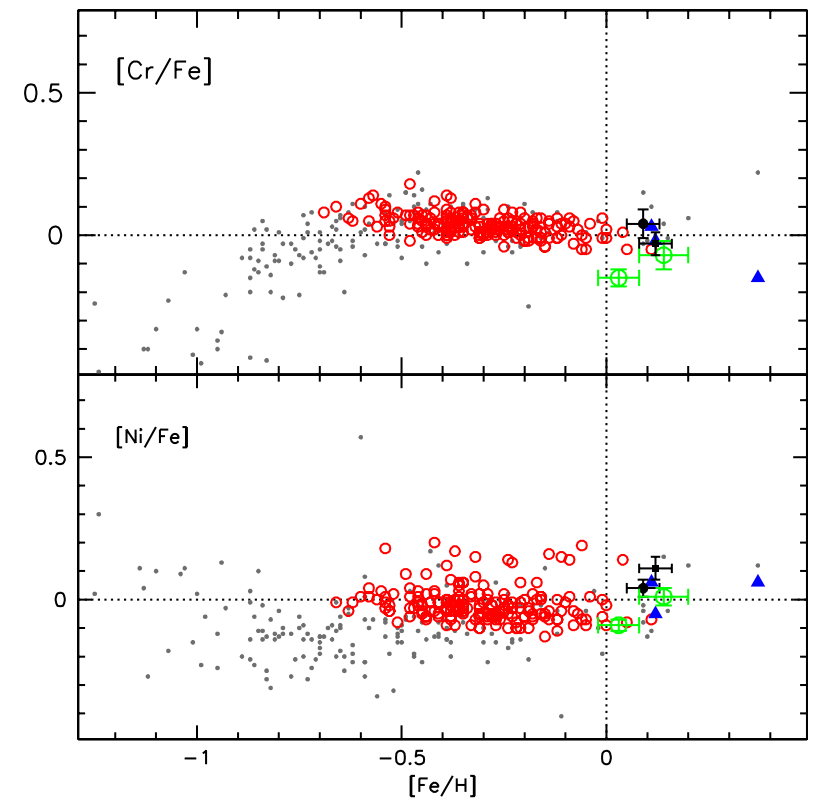

Fig. 3. Mean Ni (lower panel), and $\mathrm{Cr}$ (upper panel) abundance ratios for NGC 4337 giants (filled black squares), compared with thin-disc stars (large red open circles), thick-disc stars (grey symbols), and inner disc open clusters (Magrini et al. 2010, blue triangles), Magrini et al. (2014, green empty circles), and Trumpler 20 (black filled circle).

also considered, as in Fig. 2, old open clusters in the inner disc. The upper panel shows $\mathrm{Cr}$ abundance ratio, while the lower show Ni. Solar abundances are indicated with dotted lines.

As emphasised already in the past (Carraro et al. 2014b), the sample of Reddy et al. do not have many stars with the same metallicity as inner disc star clusters. In spite of this limitation, clusters and stars shows essentially the same trend. NGC 4337, however, shows a significant overabundance in $[\mathrm{Ni} / \mathrm{Fe}]$, compared to other clusters. In $[\mathrm{Cr} / \mathrm{Fe}]$, lastly, it shows a basically solar abundance.

\subsection{Neutron-capture elements}

There currently is a lively discussion on the $\mathrm{Ba}$ and the $\mathrm{Y}$ overabundance in star clusters and associations and its origin.

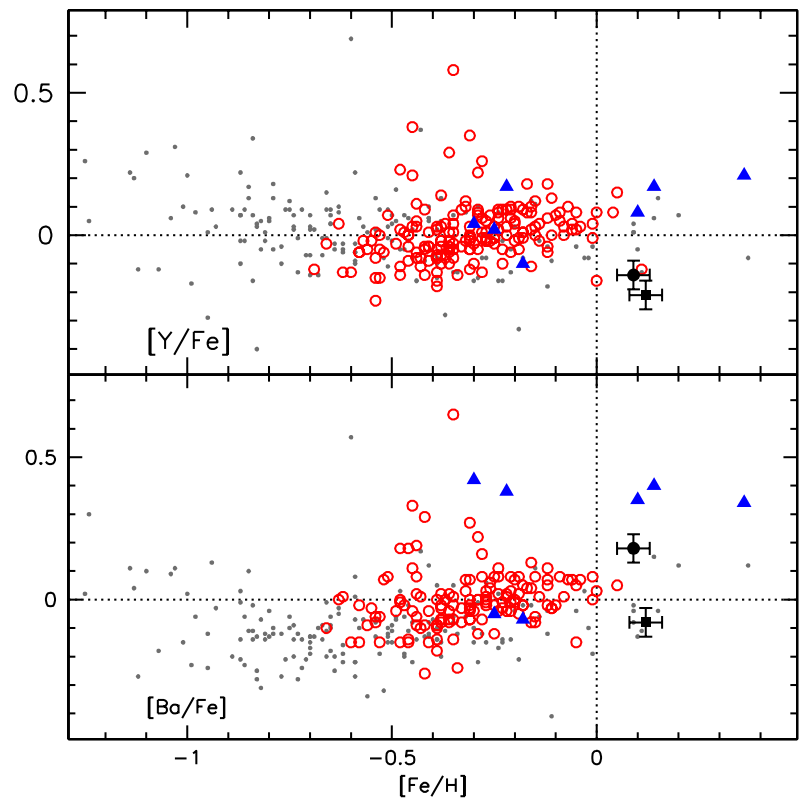

Fig. 4. Mean $\mathrm{Ba}$ (lower panel) and $\mathrm{Y}$ (upper panel) abundance ratios for NGC 4337 giants (filled black squares), compared with thin-disc stars (large red open circles), thick-disc stars (grey symbols), and old open clusters (blue triangles, from Mishenina et al. 2013). Lastly, the filled black circle indicate Trumpler 20.

According to recent studies (Mishenina et al. 2013), star clusters for which $\mathrm{Ba}$ and $\mathrm{Y}$ were measured tend to exhibit a $\mathrm{Ba}$ overabundance with respect to the Sun, and this overabundance correlates with age, namely it increases at decreasing age. In addition, for clusters younger than 3-4 Gyr, the Ba abundance shows a large scatter.

Our results are shown in Fig. 4, where NGC 4337 is compared with Trumpler 20 and the sample of old open clusters from Mishenina et al. (2013). Thin- and thick-disc stars from Reddy et al. (2003, 2006) are also plotted. Both NGC 4337 and Trumpler 20 are underabundant in Y, and are the only open clusters known to shown such underabundance above solar metallicity. NGC 4337 appears to be Ba underabundant with respect to the Sun. This behaviour is unique among open clusters more metal-rich than the Sun. 
Table 4. Mean NGC 4337 abundance ratios.

\begin{tabular}{lrc}
\hline \hline Abundance ratio & \multicolumn{1}{c}{ Mean } & $\sigma$ \\
\hline$\langle[\mathrm{C} / \mathrm{Fe}]\rangle$ & $-0.15 \pm 0.04$ & $0.02 \pm 0.01$ \\
$\langle[\mathrm{~N} / \mathrm{Fe}]\rangle$ & $0.31 \pm 0.02$ & $0.03 \pm 0.01$ \\
$\langle[\mathrm{O} / \mathrm{Fe}]\rangle$ & $-0.05 \pm 0.02$ & $0.05 \pm 0.01$ \\
$\langle[\mathrm{CNO} / \mathrm{Fe}]\rangle$ & $0.04 \pm 0.03$ & $0.03 \pm 0.01$ \\
$\langle[\mathrm{Na} / \mathrm{Fe}]\rangle$ & $0.34 \pm 0.03$ & $0.02 \pm 0.01$ \\
$\langle[\mathrm{Mg} / \mathrm{Fe}]\rangle$ & $0.06 \pm 0.04$ & $0.04 \pm 0.01$ \\
$\langle[\mathrm{Al} / \mathrm{Fe}]\rangle$ & $0.16 \pm 0.02$ & $0.03 \pm 0.01$ \\
$\langle[\mathrm{Si} / \mathrm{Fe}]\rangle$ & $0.09 \pm 0.03$ & $0.03 \pm 0.01$ \\
$\langle[\mathrm{Ca} / \mathrm{Fe}]\rangle$ & $-0.03 \pm 0.04$ & $0.02 \pm 0.01$ \\
$\langle[\mathrm{Ti} / \mathrm{Fe}]\rangle$ & $-0.10 \pm 0.03$ & $0.04 \pm 0.01$ \\
$\langle[\mathrm{alph} / \mathrm{Fe}]\rangle$ & $-0.01 \pm 0.03$ & $0.01 \pm 0.00$ \\
$\langle[\mathrm{~V} / \mathrm{Fe}]\rangle$ & $0.56 \pm 0.07$ & $0.05 \pm 0.01$ \\
$\langle[\mathrm{Cr} / \mathrm{Fe}]\rangle$ & $-0.03 \pm 0.03$ & $0.03 \pm 0.01$ \\
$\langle[\mathrm{Fe} / \mathrm{H}]\rangle$ & $0.12 \pm 0.05$ & $0.02 \pm 0.00$ \\
$\langle[\mathrm{Co} / \mathrm{Fe}]\rangle$ & $0.25 \pm 0.03$ & $0.04 \pm 0.01$ \\
$\langle[\mathrm{Ni} / \mathrm{Fe}]\rangle$ & $0.11 \pm 0.03$ & $0.02 \pm 0.00$ \\
$\langle[\mathrm{Cu} / \mathrm{Fe}]\rangle$ & $0.11 \pm 0.02$ & $0.04 \pm 0.01$ \\
$\langle[\mathrm{Zn} / \mathrm{Fe}]\rangle$ & $-0.40 \pm 0.03$ & $0.06 \pm 0.02$ \\
$\langle[\mathrm{Y} / \mathrm{Fe}]\rangle$ & $-0.21 \pm 0.03$ & $0.09 \pm 0.02$ \\
$\langle[\mathrm{Zr} / \mathrm{Fe}]\rangle$ & $-0.38 \pm 0.04$ & $0.08 \pm 0.03$ \\
$\langle[\mathrm{Ba} / \mathrm{Fe}]\rangle$ & $-0.08 \pm 0.02$ & $0.05 \pm 0.01$ \\
$\langle[\mathrm{Ce} / \mathrm{Fe}]\rangle$ & $-0.26 \pm 0.03$ & $0.06 \pm 0.02$ \\
$\langle[\mathrm{Eu} / \mathrm{Fe}]\rangle$ & $0.09 \pm 0.02$ & $0.06 \pm 0.02$ \\
\hline
\end{tabular}

\section{Fundamental parameters of NGC 4337}

We derive in this section updated estimates of the basic parameters of NGC 4337 based on the results of the previous section. To this aim we generate isochrones using the stellar models from the Padova group (Bressan et al. 2012) by adopting a metal content $[\mathrm{Fe} / \mathrm{H}]=0.12 \pm 0.05$, which translates into $Z=0.025$. We generated several isochrones, and obtained the best fit for an age of $1.6 \pm 0.1$ Gyr. The quality of the fit was judged by visually inspecting the CMD, with particular attention on the main sequence (MS) turn-off (TO) region, the MS slope, and the red clump. The best fit is shown in Fig. 5.

As a result, we estimate the reddening towards NGC 4337 to be $E(B-V)=0.23 \pm 0.05$, and its apparent distance modulus $(m-M)_{V}=12.80 \pm 0.15$. This implies a heliocentric distance of $2.6 \pm 0.2 \mathrm{kpc}$, which places the cluster at $200 \mathrm{pc}$ above the Galactic plane and $7.6 \mathrm{kpc}$ from the Galactic centre.

Having a more solid handle on the cluster metallicity and reddening, we re-compute - as an internal sanity check - the stars' $T_{\text {eff }}$ using Alonso et al. (1999) calibrations for $(B-V)$ colours. We find that the differences in $T_{\text {eff }}$ do not exceed $80 \mathrm{~K}$, and are in most case smaller than $20 \mathrm{~K}$. This is because the reddening adopted to derive $T_{\text {eff }}$ estimates in Table 2 is only 0.03 mag larger (0.26, Carraro et al. 2014a) than the reddening estimated in this paper, and the metallicity only marginally super-solar.

In Fig. 6 we show an updated version of the radial metallicity gradient in the inner disc. Differently to Carraro et al. (2014b) we added NGC 4337 (solid square) and NGC 6705 and NGC 4815 from Magrini et al. (2014, red symbols). NGC 4337 follows the trend defined by Magrini et al. (2010) sample, and Trumpler 20 very well. Including of NGC 4815 and NGC 6705, which clearly seem do deviate from the main relation, significantly decreases the slope of the gradient to $\mathrm{d}[\mathrm{Fe} / \mathrm{H}] / \mathrm{d} R=-0.12 \pm 0.10 \mathrm{dex} / \mathrm{kpc}$, and increases the metallicity scatter at any distance. We caution, however, that these two clusters are significantly younger. Lastly, we note that an almost flat gradient would result if the two most metal-rich clusters, namely NGC 6583 and NGC 6253, were removed.

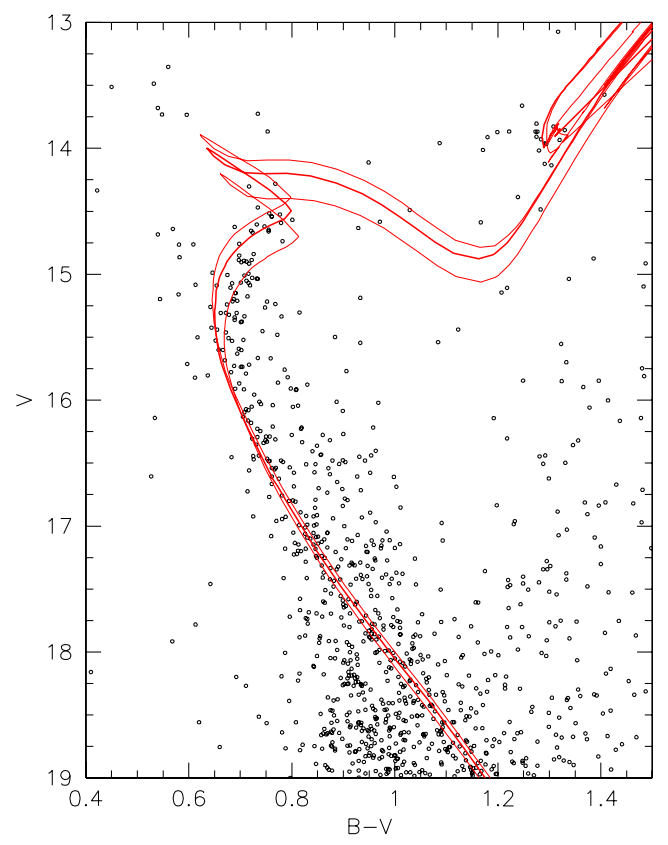

Fig. 5. $V / B-V$ CMD of NGC 4337 stars within 5 arcmin from the cluster centre. Super-imposed (red line) are $Z=0.025$ metallicity isochrones for the ages of $1.5,1.6$, and 1.7 Gyr. The inferred reddening and apparent distance modulus are $E(B-V)=0.23 \pm 0.05$ and $(m-M)_{V}=12.80 \pm 0.15$.

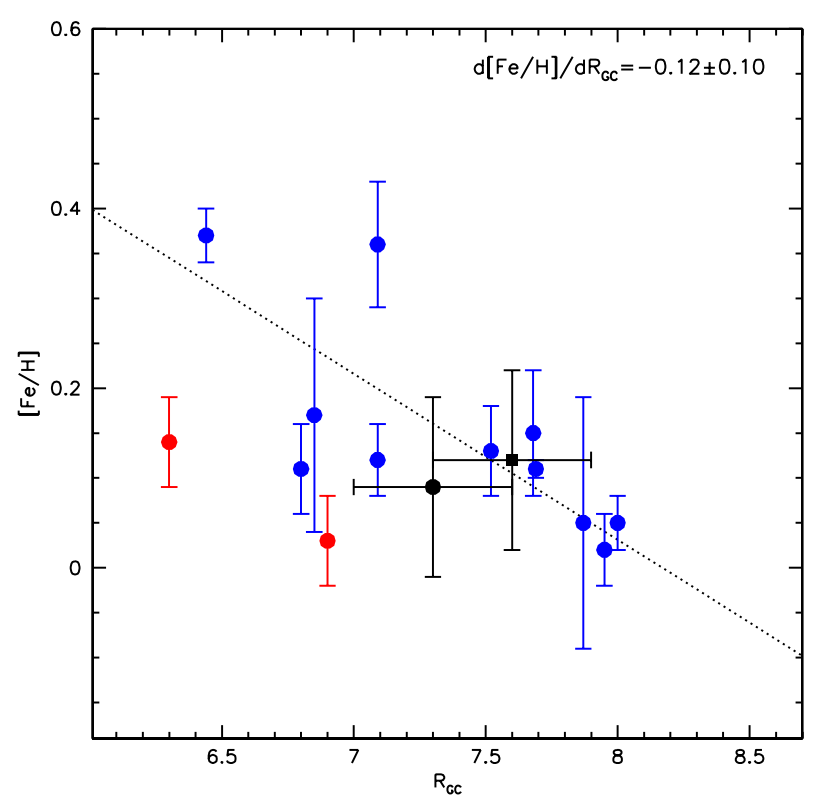

Fig. 6. Inner disc radial abundance gradient from Magrini et al. (2010, 2014; blue and red symbols, respectively) with the addition of Trumpler 20 (black circle) and NGC 4337 (black square). The dotted line is a weighted least square fit to the points, that yields -0.12 as value of the slope.

\section{Discussion and conclusions}

We have presented the first high-resolution spectroscopic study of the inner disc, old open cluster NGC 4337. Our main results are as follows:

(i) NGC 4337 has a marginally super-solar metal content $([\mathrm{Fe} / \mathrm{H}]=0.12 \pm 0.05)-$ the $\alpha$-elements are essentially solar, while we detect a significant underabundance of $\mathrm{Ba}$, unexpected in a star cluster with this metallicity. $\mathrm{Y}$ is also 


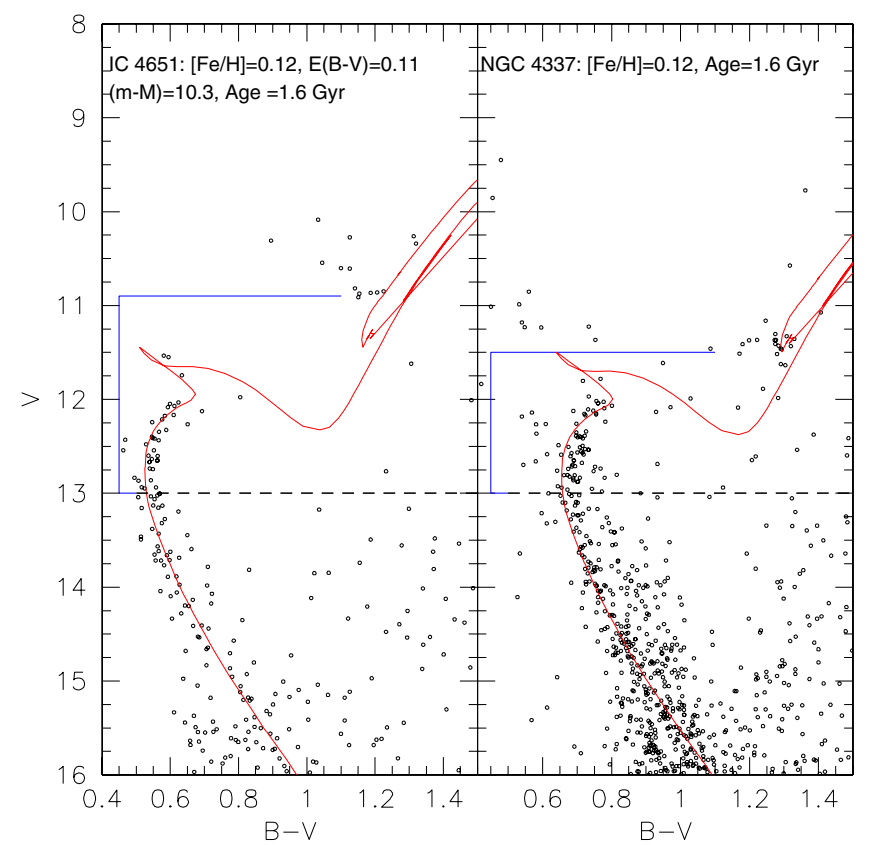

Fig. 7. Comparison of NGC 4337 (right panel) with IC 4651 (left panel). The dashed line indicates the TO magnitude of IC 4651, which NGC 4337 has been shifted to for comparison purposes. The blue segment shows the estimate of the parameter $\Delta V$. Best-fit values for the clusters' fundamental parameters are written in the top of the panels.

underabundant, similarly to Trumpler 20, and at odds with other clusters;

(ii) we refine the results of Carraro et al. (2014a), and estimate an age of $1.6 \pm 0.1 \mathrm{Gyr}$ for the cluster.

(iii) with this metallicity, and a Galacto-centric distance of $7.6 \mathrm{kpc}$, NGC 4337 fits in the inner disc radial metallicity gradient very well.

Carraro et al. (2014a) compared NGC 4337 with IC 4651, a wellknown old open cluster, and suggested that NGC 4337 is slightly younger than IC 4651, basing on the CMD morphology and the assumption of identical metallicity. The comparison was very qualitative, since it was based on an averaged IC 4651 ridgeline, which is easy to draw for the MS, but can be much more complicated for the clump, which, for this age range, is typically quite broad, both in colour and in magnitude.

Now that we independently estimate the age and metallicity of NGC 4337, we can compare it again with IC 4651. According to Anthony-Twarog et al. 2009), IC 4651 is 1.5 Gyr old for a metallicity $[\mathrm{Fe} / \mathrm{H}]=0.12$ (see also Carretta et al. 2004), identical to the metallicity of NGC 4337 . It has a reddening $E(B-V) \sim$ 0.12 , and an apparent distance modulus $(m-M) \sim 10.40$.

We compare the two clusters in Fig. 7. The blue segment shows the estimate of the parameter $\Delta V$, defined as the magnitude difference between the blue TO and the red clump magnitude. This parameter is a very well-known relative age indicator (Carraro \& Chiosi 1994), independent on reddening, and with some dependence on metallicity. A higher $\Delta V$ values means an older age. In this case, the $\Delta V$ for IC 4651 and NGC 4337 are 2.1 and $1.6 \mathrm{mag}$.

Super-imposed on the NGC 4337 stars is the best-fit isochrone discussed in Sect. 5. Since NGC 4337 and IC 4651 have the very same metallicity, we super-imposed the same isochrone on top of IC 4651. A $1.6 \mathrm{Gyr}$ isochrone misses the clump, which is clearly brighter and at the same time does not reproduce the TO curvature and extension well.

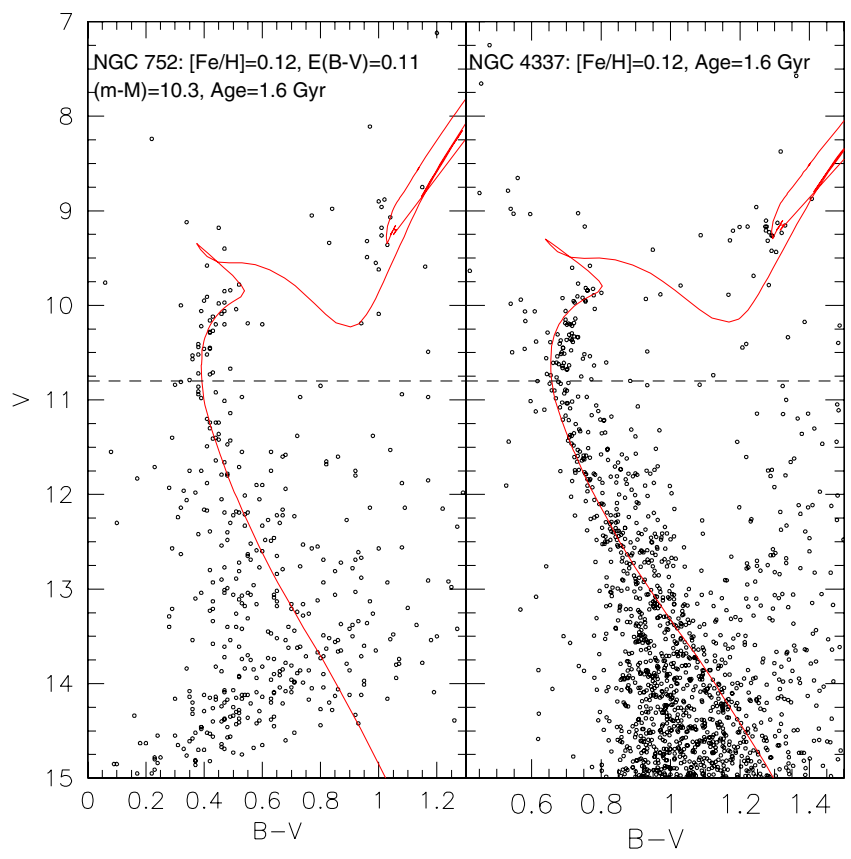

Fig. 8. Comparison of NGC 4337 (right panel) with NGC 752 (left panel). The dashed line indicates the TO magnitude of NGC 752, which NGC 4337 has been shifted to for comparison purposes. Best-fit values for the clusters' fundamental parameters are written in the top of the panels.

This evidence, in tandem with the higher value of $\Delta V$, suggests that IC 4651 is significantly older than NGC 4337. In our scale (the Padova models), IC 4651 would in fact be about 1.9 Gyr old. This age would be consistent with a lower reddening $E(B-V) \sim 0.09 \mathrm{mag}$, and a smaller apparent distance modulus $(m-M)_{V} \sim 10.15 \mathrm{mag}$.

Therefore, although they share the same metallicity, NGC 4337 and IC 4651 do have significantly different ages.

We found NGC 752 (Anthony-Twarog \& Twarog 2006) to be a stronger case for a NGC 4337 twin. NGC 752 has a marginally lower metallicity $([\mathrm{Fe} / \mathrm{H}]=0.08 \pm 0.04$, Carrera \& Pancino 2011) which appears to be very similar to NGC 4337 one within the uncertainties, however.

The comparison is illustrated in Fig. 8, where the CMD of NGC 4337 is compared to the CMD of NGC 752 from Francic (1989). We adopted for both cluster the same metallicity $([\mathrm{Fe} / \mathrm{H}]=0.012)$ and the same age $(1.6 \mathrm{Gyr})$. The fit is remarkably good in both cases, strongly suggesting that these two clusters share the same chemical and evolutionary phase.

While the clump in both clusters is equally populated and possibly delineates the same evolutionary phase (Girardi et al. 2000), the real difference among these two clusters is in the MS, which for NGC 752 shows a significant star-smearing immediately below the TO. It is indeed impossible to follow the cluster MS below $V \sim 11.50 \mathrm{mag}$. This is interpreted as a signature that NGC 752 has undergone quite a strong dynamical evolution and is on the verge of dissolving into the general Galactic field. Since the two clusters are coeval, this suggests that either NGC 4337 formed as a more massive cluster than NGC 752, or that the latter experienced a much stronger tidal interaction with the Milky Way than the former.

Because it is more massive, and hence has retained more stars, NGC 4337 is an ideal object in which to compare the star distribution in the CMD against stellar evolution models in a statistical manner. 
Acknowledgements. S. Villanova gratefully acknowledges the support provided by FONDECYT program N. 1130721. G. Carraro expresses his gratitude to B. Twarog for his very useful comments. The ESO Director General is deeply thanked for granting time to this project via the Director General Discretionary Time (DDT).

\section{References}

Alonso, A., Arribas, S., \& Martínez-Roger, C. 1999, A\&AS, 140, 261 Anthony-Twarog, B. J., \& Twarog, B. A. 2006, PASP, 118, 358

Anthony-Twarog, B. J., Mukherjee, K. Caldwell, N., \& Twarog, B. A. 1988, AJ, 95,1453

Anthony-Twarog, B. J., Delyinannis, C. P., Twarog, B. A., Croxall, K. W., \& Cummings, J. D. 2009, AJ, 138, 1171

Bensby, T., Alves-Brito, A., Oey, M. S., Yong, D., \& Meléndez, J. 2010, A\&A, 516, L13

Bertelli, G., Bressan, A., \& Chiosi, C. 1992, ApJ, 392, 522

Bressan, A., Marigo, P., Girardi, L., et al. 2012, MNRAS, 427, 127

Carraro, G., \& Chiosi, C. 1994, A\&A, 287, 761

Carraro, G., Bertelli, G., Bressan, A., \& Chiosi, C. 1993, A\&AS, 101, 301

Carraro, G., Chiosi, C., Bressan, A., \& Bertelli, G. 1994, A\&AS, 103, 375

Carraro, G., Giorgi, E. E., Costa, E., \& Vázquez, R. A. 2014a, MNRAS, 441, L36

Carraro, G., Villanova, S., Monaco, L., et al. 2014b, A\&A, 562, A39
Carrera, R., \& Pancino, E. 2012, A\&A, 535, 30

Carretta, E., Bragaglia, A., Gratton, R. G., \& Tosi, M. 2004, A\&A, 422, 951

Coelho, P., Barbuy, B., Meléndez, J., Schiavon, R. P., \& Castilho, B. V. 2005, A\&A, 443, 735

Francic, S. P. 1989, AJ, 98, 888

Froebrich, D., Schmeja, S., Samuel, D., \& Lucas, P. W. 2010, MNRAS, 409, 1281

Grevesse, N., \& Sauval, A. J. 1998, SSRv, 85, 161

Girardi, L., Mermilliod, J.-C., \& Carraro, G. 2000, A\&A, 359, 892

Kurucz, R. L. 1970, in SAO Special Report (Cambridge: Smithsonian Astrophysical Observatory), 309

Kurucz, R. L., Furenlid, I., Brault, J., \& Testerman, L. 1984, Solar flux atlas from 296 to $1300 \mathrm{~nm}$ (National Solar Observatory)

Magrini, L., Randich, S., Zoccali, M., et al. 2010, A\&A, 523, A11

Magrini, L., Randich, S., Romano, D., et al. 2014, A\&A, 563, A44

Marino, A. F., Villanova, S., Piotto, G., et al. 2008, A\&A, 490, 625

McWilliam, A. 1998, AJ, 115, 1640

McWilliam, A., \& Rich, R. M. 1994, ApJS, 91, 749

Mishenina, T., Korotin, S., Carraro, G., Kovtyukh, V. V., \& Yegorova, I. A. 2013, MNRAS, 433, 1436

Reddy, B. E., Tomkin, J., Lambert, D. L., \& Allende Prieto, C. 2003, MNRAS, 340,304

Reddy, B. E., Lambert, D. L., \& Allende Prieto, C. 2006, MNRAS, 367, 1329

Sneden, C. 1973, ApJ, 184, 839

Villanova, S., Carraro, G., \& Saviane, I. 2009, A\&A, 504, 845 